\title{
Centrifugally driven convection in the rotating cylindrical annulus with modulated boundaries
}

\author{
M. Westerburg and F. H. Busse \\ Institute of Physics, University of Bayreuth, D-95440 Bayreuth, Germany \\ Received: 18 June 2002 - Revised: 17 October 2002 - Accepted: 21 October 2002
}

\begin{abstract}
The effect of sinusoidally modulated conical end boundaries on convection in a rotating cylindrical annulus is investigated theoretically and experimentally. A quasiperiodic time dependence of convection in the form of thermal Rossby waves is found and semi-quantitative agreement between theory and measurements can be established. The results are relevant to convection in the Earth's outer core close to the tangent cylinder touching the inner core at its equator.
\end{abstract}

\section{Introduction}

Convection driven by centrifugal buoyancy in a rotating cylindrical annulus has become one of the basic fluid dynamical systems in which the interactions between buoyancy driven flows and rotation can be studied in their simplest realizations. In particular, the annulus model exhibits all basic dynamical features of convection in rotating spherical shells which represents a fundamental problem in astrophysics and planetary science. The rotating annulus system can easily be realized in the laboratory (Busse and Carrigan, 1974) and several experimental studies have been performed (Azouni et al., 1986; Busse et al., 1997). Among the theoretical investigations we mention only some recent ones (Schnaubelt and Busse, 1992, 1997), in which realistic no-slip boundary conditions have been used.

While the annulus system as well as the spherical shell are usually considered as axisymmetric systems the influence of non-axisymmetric modulations of the boundaries has received much attention in recent years. In the geophysical context the effects of bumps on the core-mantle boundary of the Earth have been considered for a long time (Hide, 1989) and have motivated the recent analysis by Bassom and Soward (1996) and Soward and Bassom (1997). The analogous problem for the rotating cylindrical annulus has been treated by Bell and Soward (1996, see also Bell, 1993) and

Correspondence to: M. Westerburg

(Michael.Westburg@uni-bayreuth.de) by Herrmann and Busse (1998). In the present paper an experimental investigation of the modulated annulus problem will be reported. Since the previous theoretical studies have been done for a parameter range that is not easily accessible in the laboratory, we also shall describe some numerical computations which show a close correspondence with the experimentally observed phenomena.

The problem of convection in the presence of a spatial modulation is also of interest from a basic physical point of view. Numerous papers have been published on the effect of modulated boundary conditions on Rayleigh-Bénard convection in a horizontal fluid layer heated from below (Kelly and Pal, 1978; Pal and Kelly, 1978; Hartung et al., 1990, 1991; Zimmermann et al., 1998; and others referred to in these papers). The analysis of the Rayleigh-Bénard problem in the case of sinusoidal variations of the height of the layer or of the temperature prescribed at the boundaries is hampered by the fact that the basic state is no longer a motionless state. This property, however, can be preserved in the case of centrifugally driven convection in the rotating cylindrical annulus. The latter problem is also richer than the problem of modulated Rayleigh-Bènard convection because convection assumes the form of traveling thermal Rossby waves. There are thus various kinds of time dependent forms of convection besides the steady mode locked to the modulated boundary.

The rotating cylindrical annulus on which the attention will be focused is shown in Fig. 1. Convection is driven by centrifugal buoyancy in the fluid layer between the cooled inner cylinder and the heated outer cylinder. The Coriolis force enters the dynamics of convection primarily through the effect of the conical end boundaries of the annular gap. Even in the axisymmetric case when the height $h$ of the gap varies with distance from the axis it prevents the realization of purely two-dimensional convection rolls aligned with the axis. Without this change in height steady two-dimensional rolls would be possible solutions of the basic equations and the Coriolis force would be balanced entirely by the pressure gradient. But owing to the change in height the convection rolls or columns assume the dynamical properties of 


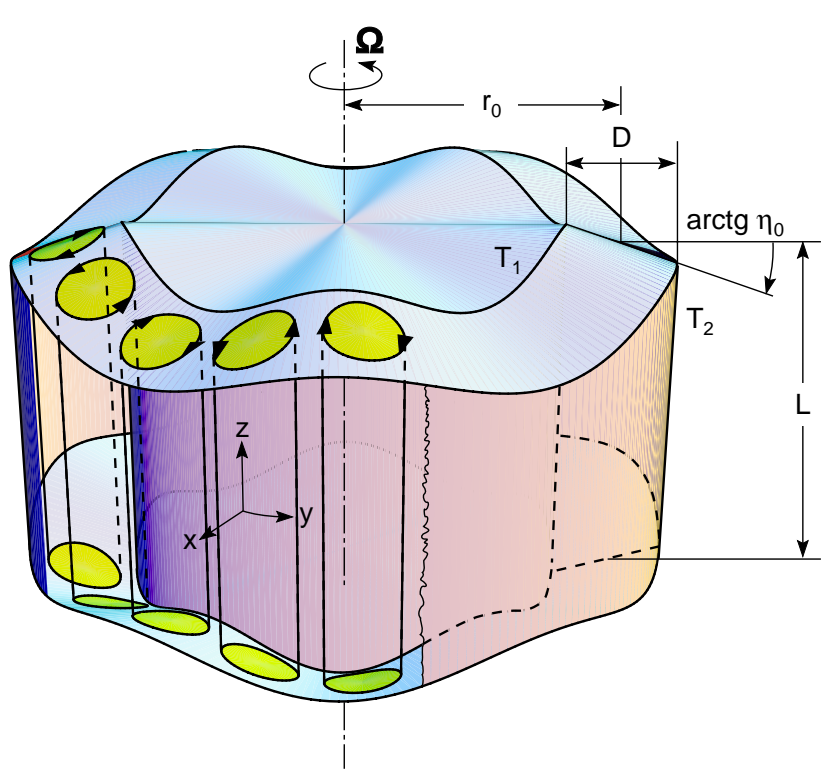

Fig. 1. Geometrical configuration of the rotating modulated cylindrical annulus.

Rossby waves and propagate in the azimuthal direction given by $\nabla h \times \boldsymbol{\Omega}$ where $\boldsymbol{\Omega}$ is the rotation vector of the system. A modulation of these waves occurs when the height varies also in the azimuthal direction as indicated in Fig. 1. This modulation breaks the azimuthal symmetry, but it still admits a motionless basic state. This feature simplifies the analysis of the convection flow and also makes the system attractive from the experimental point of view.

In the following we shall briefly introduce the theoretical description of the problem in Sect. 2 and describe some results of the linear analysis for the onset of convection in Sect. 3. The nonlinear properties of convection in the form of propagating thermal Rossby waves are discussed in Sect. 4. In Sect. 5 the laboratory apparatus and the measurement techniques are briefly described and experimental results are presented. A concluding discussion is given in the last section of the paper.

\section{Mathematical formulation of the problem}

We consider the modulated cylindrical annulus of average height $L$ as shown in Fig. 1. The temperatures $T_{1}$ and $T_{2}$ are prescribed on the inner and outer cylinders, respectively, and the entire configuration is rotating rigidly with the angular velocity $\Omega$ about its axis. Using the gap width $D$ as length scale and $D^{2} / v$ as time scale where $v$ is the kinematic viscosity of the fluid, we arrive at the following system of equations for the stream function $\psi$ and the deviation $\Theta$ of the temperature from the static state of a purely conducting profile,

$$
\left(\frac{\partial}{\partial t}+\frac{\partial}{\partial y} \psi \frac{\partial}{\partial x}-\frac{\partial}{\partial x} \psi \frac{\partial}{\partial y}-\Delta_{2}\right) \Delta_{2} \psi-\eta
$$

$$
\begin{aligned}
& \left(1+\delta \cos \alpha_{M} y\right) \frac{\partial}{\partial y} \psi+\epsilon \sin \alpha_{M} y \frac{\partial}{\partial x} \psi+R \frac{\partial}{\partial y} \Theta=0 \\
& P\left(\frac{\partial}{\partial t}+\frac{\partial}{\partial y} \psi \frac{\partial}{\partial x}-\frac{\partial}{\partial x} \psi \frac{\partial}{\partial y}\right) \Theta-\Delta_{2} \Theta+\frac{\partial}{\partial y} \psi=0
\end{aligned}
$$

where $\Delta_{2} \equiv \partial^{2} / \partial x^{2}+\partial^{2} / \partial y^{2}$ and where the Rayleigh number $R$, the Prandtl number $P$, the Coriolis parameter $\eta$ and the dimensionless modulation parameters $\delta$ and $\varepsilon$ are defined by

$$
\begin{aligned}
& R=\frac{\gamma\left(T_{2}-T_{1}\right) \Omega^{2} r_{0} D^{3}}{\nu \kappa}, \quad P=\frac{\nu}{\kappa}, \quad \eta=\frac{4 \eta_{0} \Omega D^{3}}{\nu L}, \\
& \delta=\frac{2 \hat{\epsilon} D}{L}, \quad \epsilon=\frac{4 \hat{\epsilon} \alpha_{M} \Omega D^{3}}{\nu L}
\end{aligned}
$$

Here $\gamma$ is the coefficient of thermal expansion, $\kappa$ is the thermal diffusivity and $r_{0}$ is the mean radius of the annulus. $\alpha_{M}$ is the wavenumber of the modulation as defined in expression (3) below. A Cartesian coordinate system has been introduced with the $x, y$ and $z$-coordinates oriented in the radial, azimuthal and axial directions, respectively. The terms involving $\eta, \delta$ and $\epsilon$ arise when the equation for the $z$ component of vorticity, $\Delta_{2} \psi$, is averaged over the height of the annulus and the condition that the normal component of the velocity vanishes at the modulated boundaries

$z_{ \pm}= \pm \frac{L}{2 D} \mp \eta_{0} x \mp \hat{\epsilon} \cos \alpha_{M} y$

has been taken into account together with the assumptions $\eta_{0} \ll 1, \hat{\epsilon} \ll L / D$. Through this procedure it has been possible to reduce the original equations for the threedimensional velocity field $\left(v_{x}, v_{y}, v_{z}\right)$ to an equation for its dominating two-dimensional geostrophic component, $v_{x}=$ $\partial \psi / \partial y, v_{y}=-\partial \psi / \partial x$, described by the stream function $\psi$. For a more detailed discussion of the problem without modulation we refer to Busse (1986). The problem with modulation has been treated in the limit $\delta=0$ by Herrmann and Busse (1998), which will be referred to by HB98 in the following. An earlier analysis of the problem based on periodic boundary conditions in the $x$-direction has been given by Bell and Soward (1996; see also Bell, 1993). In the present analysis we shall focus the attention on the case $\epsilon=0, \delta \neq 0$ which has not been considered previously, but is close to the experimental situation.

In order to solve Eqs. (1) in the experimentally realistic case of no-slip cylindrical boundaries at which the conditions

$\frac{\partial}{\partial y} \psi=\frac{\partial}{\partial x} \psi=\Theta=0$ at $x= \pm \frac{1}{2}$

are imposed, we introduce the Galerkin representation

$$
\begin{aligned}
& \psi=\sum_{l=1, n=0}^{\infty}\left(\hat{a}_{l n}(t) \cos n \alpha y+\check{a}_{l n}(t) \sin n \alpha y\right) \\
& f_{l}(x)+\left(4 x^{3}-3 x\right) \hat{a}_{00}(t) \\
& \Theta=\sum_{l, n}\left(\hat{b}_{l n}(t) \cos n \alpha y+\check{b}_{l n}(t) \sin n \alpha y\right) \sin l \pi\left(x+\frac{1}{2}\right)
\end{aligned}
$$


where the functions $f_{l}(x)$ denote the Chandrasekhar functions

$f_{l}=\left\{\begin{array}{l}\frac{\cosh \lambda_{l} x}{\cosh \lambda_{l} \frac{1}{2}}-\frac{\cos \lambda_{l} x}{\cos \lambda_{l} \frac{1}{2}} \text { for } l=\text { odd } \\ \frac{\sinh \lambda_{l} x}{\sinh \lambda_{l} \frac{1}{2}}-\frac{\sin \lambda_{l} x}{\sin \lambda_{l} \frac{1}{2}} \text { for } l=\text { even }\end{array}\right.$

The numbers $\lambda_{l}$ are obtained as the roots of the equations

$\tanh \lambda_{l} \frac{1}{2}+\tan \lambda_{l} \frac{1}{2}=0$ for $l=$ odd,

$\operatorname{coth} \lambda_{l} \frac{1}{2}-\cot \lambda_{l} \frac{1}{2}=0$ for $l=$ even

By adding the last term on the right hand side of expression (5a) we have accommodated the fact that a vanishing zonal mass flux through the annulus cannot be required and thus the condition $\psi=0$ at $x= \pm \frac{1}{2}$ must not be used for the $y$-independent part of $\psi$. Instead the consequence of the absence of a mean pressure gradient in the azimuthal direction must be considered. This latter condition is satisfied when $\hat{a}_{00}(t)$ obeys the equation

$\frac{\partial}{\partial t} \hat{a}_{00}(t)=-\sum_{l=\text { even }} \hat{a}_{l 0}(t) f_{l}^{\prime \prime}\left(\frac{1}{2}\right)-12 \hat{a}_{00}(t)$

After expressions (5) have been introduced in Eq. (6) and these have been projected onto the sets of expansion functions used in Eq. (5), a system of nonlinear differential equations in time are obtained for the unknown coefficients $\hat{a}_{l n}(t), \check{a}_{l n}(t), \hat{b}_{l n}(t), \breve{b}_{l n}(t)$. Through numerical forward integration in time this system of equations can be solved once it has been truncated. By neglecting all coefficients and corresponding equations with subscripts $l, n$ which satisfy either one of the conditions

$l>N_{x} \quad, \quad n>N_{y}$

we obtain a finite system. A typical value used for $N_{x}$ is 6 , but much larger values up to 63 have been used for $N_{y}$ depending on the ratio between $\alpha$ and $\alpha_{M}$. Various other truncation values have been used in order to ensure that all physically relevant properties of the solutions change only insignificantly when $N_{x}$ and $N_{y}$ are replaced by $N_{x}+2,2 N_{y}+$ 1.

\section{Onset of convection}

For the determination of the onset of convection based on the linearized version of Eq. (1), we use the Floquet ${ }_{\text {ansatz }}$

$$
\begin{aligned}
& \psi=\exp \{i d y+\sigma t\} \sum_{l, n} a_{l n} \exp \left\{i n \alpha_{M} y\right\} f_{l}(z) \\
& \Theta=\exp \{i d y+\sigma t\} \sum_{l, n} b_{l n} \exp \left\{i n \alpha_{M} y\right\} \sin l \pi\left(x+\frac{1}{2}\right)
\end{aligned}
$$

The solution of the linear algebraic eigenvalue problem for the eigenvalue $\sigma$ will determine $\sigma$ as a function of $d$ for given values of $R, \delta, \alpha_{M}, \eta, P$. Whenever there exists a positive

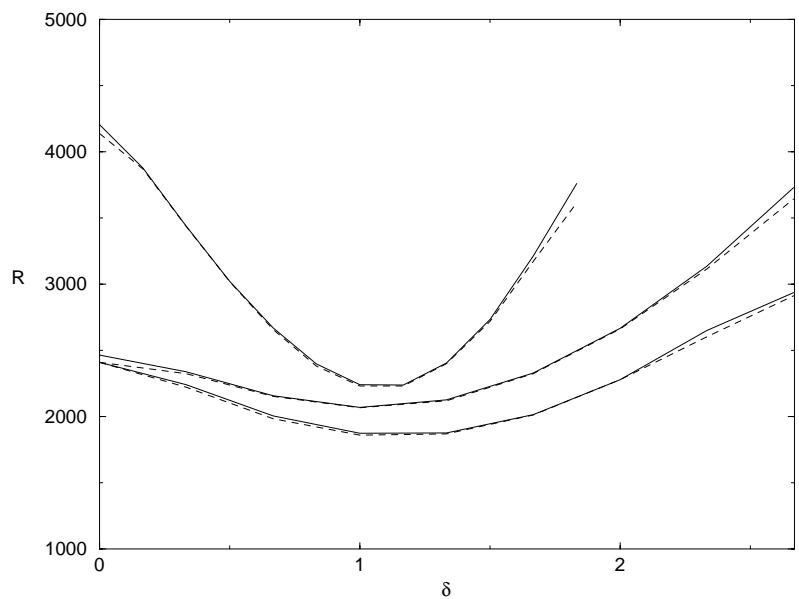

Fig. 2. Critical Rayleigh number $R_{C}$ for onset of convection as a function of $\delta$ in the cases $\eta=600$ with $\alpha_{M}=1.0, \eta=300$ with $\alpha_{M}=1.0$ and with $\alpha_{M}=0.5$ (from top to bottom). For each of the three cases the results for vanishing Floquet wavenumber, $d=0$ (solid curves) and for finite values of $d$ (dashed curves) are shown. $P=0.71$ in all cases.

real part $\sigma_{r}$ of $\sigma$, the static state of pure conduction is unstable, otherwise it will be regarded as stable. It is a demanding task to investigate the full five-dimensional parameter space spanned by the parameters $R, \delta, \alpha_{M}, \eta, P$ and we shall restrict the analysis to a few special cases.

In Fig. 2 results for the onset of convection are shown for modulations with relative long wavelengths, $\alpha_{M}=0.5$ and 1.0. The system exhibits a local response in that the onset of convection occurs where the expression $\hat{\eta}(y) \equiv$ $\eta\left(1+\delta \cos \alpha_{M} y\right)$ reaches a minimum. The critical Rayleigh number $R_{c}$ thus decreases with increasing $\delta$ and reaches a minimum when $\delta$ approaches unity. Beyond that point $\hat{\eta}(y)$ becomes negative in finite intervals of $y$ and $R_{c}$ increases again since a zero-crossing of $\hat{\eta}(y)$ does not provide the same optimal conditions for the onset of convection as the place where $\hat{\eta}(y)$ touches zero. Since in experimental realisations only discrete wavelengths are admissible in the azimuthal direction we have indicated in Fig. 2 Rayleigh numbers for the case $d=0$ as well as for the minimizing value of $d$. Obviously there is no significant difference between the two types of curves in the case of small $\alpha_{M}$. For $d=0$, for instance, dominating coefficients in expressions (9) are found for $l=1, n=3$ in the case $\alpha_{M}=1.0$ and for $l=1, n=7$ in the case $\alpha_{M}=0.5$. When finite values of $d$ are admitted, $d \approx 0.5$ and $d \approx 0$ are found in the two cases.

When the modulation wavenumber becomes more comparable with the typical wavenumber $\alpha$ of convection the modulation exerts a stabilizing influence on the onset of thermal Rossby waves as can be seen in Fig. 3. The difference between the critical Rayleigh numbers calculated with $d=0$ and with $d \neq 0$ is more significant in this case. For sufficiently high amplitudes $\delta$ of modulation a new mode of convection becomes preferred, however, which is locked to the modulation and thus steady. This mode was found by 


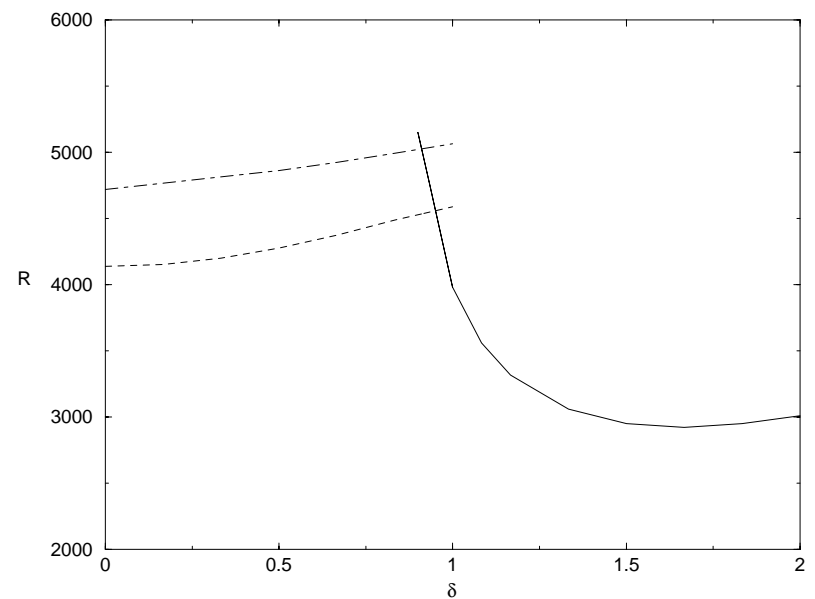

Fig. 3. Critical Rayleigh number $R_{C}$ for onset of convection as a function of $\delta$ in the case $\eta=600$ with $\alpha_{M}=3.0$ and $P=0.71$. The dotted (dash-dotted) curve corresponds to the onset of thermal Rossby waves with finite (vanishing) value of $d$. The solid curve corresponds to the steady pinned mode of convection.

Bell (1993; see also Bell and Soward, 1996) and was investigated in HB98 for realistic boundary conditions. For $\alpha_{M} \simeq 3$ the critical Rayleigh number exhibits a very wide minimum. But as $\alpha_{M}$ decreases the minimum becomes rather sharp as shown by the example of Fig. 4. For a more systematic study of this behavior see HB98. Because the emphasis of this paper is on the nonlinear aspects of the problem we shall close the consideration of the linear problem at this point and mention only that the results for the steady mode hold independently of the Prandtl number $P$ since $P$ appears only in connection with the time derivative of $\Theta$ in the linear version of Eq. (1).

\section{Nonlinear analysis of convection}

Solutions of the fully nonlinear system of Eq. (1) are obtained through the Galerkin representation (5). Since the modulation wave number $\alpha_{M}$ is usually much smaller than the natural wavenumber of the thermal Rossby waves a much higher truncation is used for the index $n$ than for the index $l$ in expressions (5) and the associated system of ordinary differential equations in time for the coefficients $\hat{a}_{l n}(t), \check{a}_{l n}(t)$, $\hat{b}_{l n}(t)$ and $\breve{b}_{l n}(t)$. Usually truncations $l \leq 6$ or 8 and $n \leq 32$ have been used as we have already mentioned above.

A typical result of the computations is shown in Fig. 5 where the evolution of the amplitude of the modulated Rossby waves is shown as a function of time. At the point $y=0$ the expression $1+\delta \cos \alpha_{M} y$ reaches its maximum and thus the inhibiting influence of rotation is especially strong. The thermal Rossby waves entering from the left thus decay in amplitude as they propagate towards the right. Because the local value of the phase velocity also reaches a maximum at $y=0$ the lines of constant phase are most strongly inclined at this point. The opposite behavior can be observed

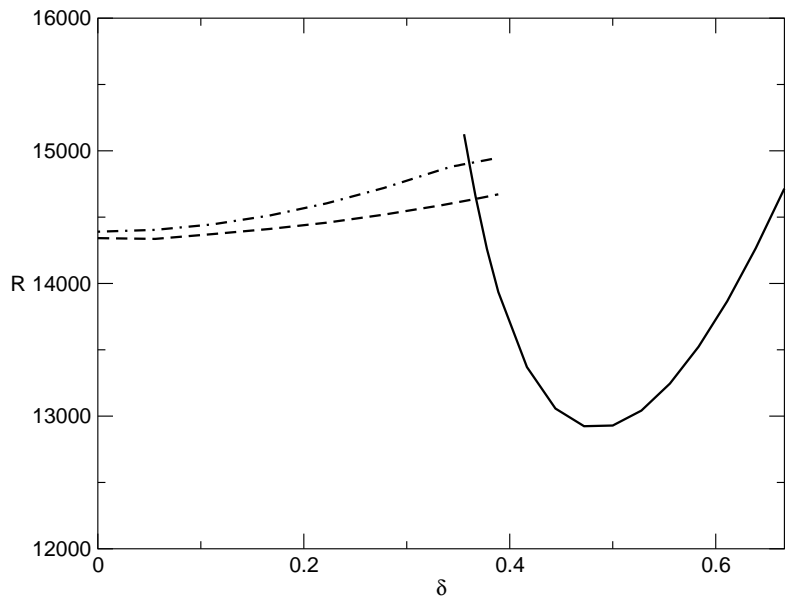

Fig. 4. Same as Fig. 3 but for $\eta=1800$ with $\alpha_{M}=1.0$.

at $y=\pi / \alpha_{M}$ where the local constraint of rotation reaches a minimum. The thermal Rossby waves thus slow down and increase in amplitude as they propagate.

For the comparison with experimental observation it is of interest to follow the time dependence of a variable, say the temperature, at a fixed point in space. In Fig. 6a typical set of traces in time are shown which demonstrate the nature of time dependence may change significantly from one point to a neighboring one. Especially at the stations where $\left|\sin \alpha_{M} y\right|$ is large such that the local value of $\eta$ changes most rapidly the time dependence is characterized by the decaying long wavelength part and the growing short wavelength part of the Rossby waves (for $\sin \alpha_{M} y<0$ ) and vice versa (for $\sin \alpha_{M} y>0$ ). The trains of Rossby waves are more regular shortly after $\cos \alpha_{M} y$ reaches its maximum or minimum.

\section{Experimental observations}

Convection driven by centrifugal buoyancy in a rotating cylindrical annulus can be realized in relatively simple ways in laboratory experiments. The action of gravity is minimized when a vertical axis of rotation is used and the rate of rotation is chosen sufficiently high such that the centrifugal force exceeds that of gravity by a factor of two or three. Reports on results from experiments with axisymmetric annulus configurations can be found in the papers by Busse et al. (1997) and references therein and by Jaletzky and Busse (2000). The drawing shown in Fig. 1 of the latter paper indicates details of the apparatus which has been modified in order to realize an annular region as indicated in Fig. 1. Two aluminum cylinders have been used to create a fluid gap of thickness $D=10.4 \mathrm{~mm}$ with a mean radius $r_{o}=70.2 \mathrm{~mm}$. Conical end boundaries with the angle $\operatorname{arctg} \eta_{0}=\pi / 4$ are introduced which are modulated in the azimuthal direction with the azimuthal wavenumber $m=2$ and with an amplitude of $D \hat{\epsilon}=9.5 \mathrm{~mm}$. The dimensionless modulation wavenumber thus is $\alpha_{M}=0.296$. The average height $L$ of the fluid 


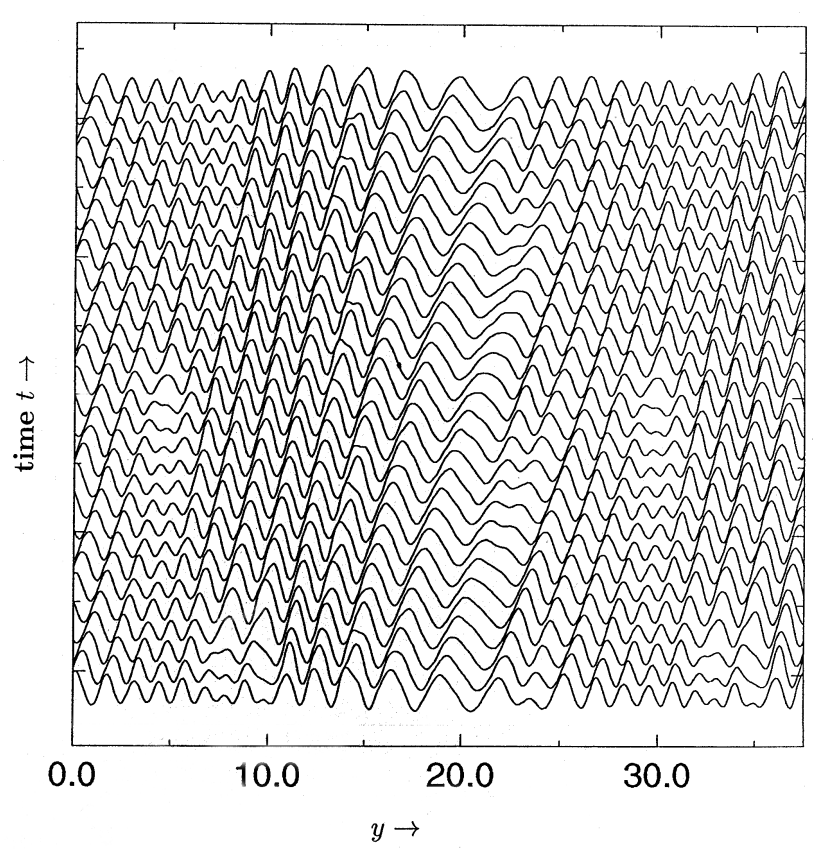

Fig. 5. The temperature $\Theta$ at the point $x=-0.4$ as a function of $y$ at different instances in time, $t=t_{0}+n \Delta t, n$ increasing from bottom to top with $\Delta t=0.06$, in the case $R=3000, \eta=300$, $\alpha_{M}=0.25, \delta=0.3, P=0.71$.

gap is kept variable. But usually relatively low values have been used in order to reach high values of $\eta$ and $\delta$ for which the modulation effects become most clearly visible. This latter goal conflicts, of course, with assumption of $\hat{\epsilon} \ll L / D$ on which the theoretical analysis is based. Discrepancies between theoretical predictions and measurements must thus be expected. Differences between theoretical calculations carried out for $\epsilon=0$ and experimental observations should also be anticipated because $\epsilon$ is not negligible in the experiment. The effect of $\epsilon$ on the dynamics of thermal Rossby waves is comparable to, though somewhat weaker than the effect of $\eta \delta$ as can be seen from a comparison of the results of Figs. 2 and 3 with those given in HB98. In the experiment $\eta \delta \approx 2 \epsilon$ holds and thus some influence of the finite value of $\epsilon$ must be expected.

The existence of thermal Rossby waves is detected by thermistors imbedded in the wall of the inner cylinder at its equatorial plane. The tiny thermistors (with a diameter of $0.41 \mathrm{~mm}$ from Bowthorpe Thermometrics) with a resistance of $10 \mathrm{k} \Omega$ at $25^{\circ} \mathrm{C}$ glued into little holes in the aluminum wall such that they reach up to a distance of $0.5 \mathrm{~mm}$ into the fluid. This distance is small enough to avoid any significant disturbance of the flow, but improves the detection of temperature variations. The resistance of the thermistors is measured by digital multimeters (PREMA) to which they are electrically connected through slip-rings. Most of the experiments have been carried out with air as fluid. More accurately it should be said that nitrogen had been used instead of air in order to avoid the problem of eliminating the water vapor from the

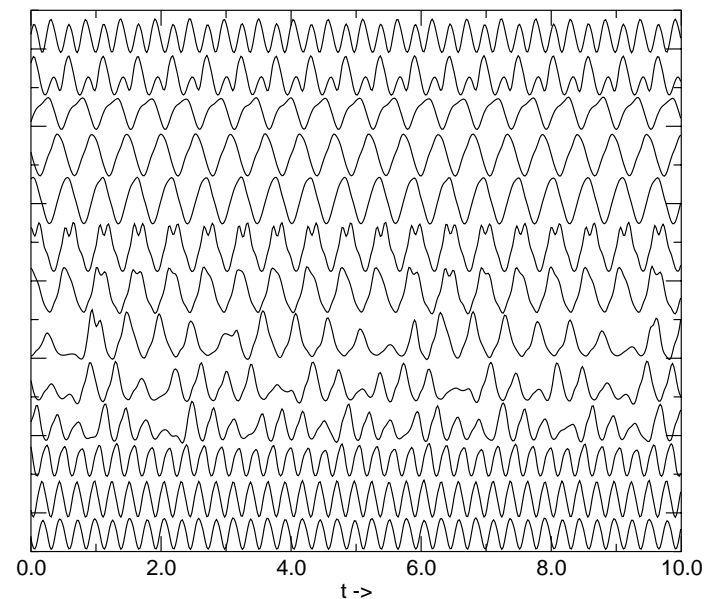

Fig. 6. Time dependence of the temperature $\Theta(x, y, t)$ at $x=-0.4$ and at the values $y=2 \cdot m$ with $m=0,1, \ldots, 12$ (from bottom to top) in the case $R=4000, \eta=300, \alpha_{M}=0.25, \delta=0.4, P=$ 0.71 .

air. Since the properties of nitrogen and dry air are nearly the same we shall neglect the differences between the two fluids. Some experiments have also been carried out with water.

Examples of the thermistor records are shown in Fig. 7. These records have been high-pass filtered since slight variations in the temperatures of the thermostats caused long period variations of the order of several $10^{-2} \mathrm{~K}$ in the temperatures of the aluminum walls. The thermistor records of Fig. 7 clearly demonstrate how sensitively the structure of the signal depends on the position of the thermistor. The values of the Rayleigh number referred to in the figure caption represent overestimates since the heat transport provided by convection has not been taken into account in calculating the temperature difference between the walls.

As mentioned before the assumptions of the theory are not well approximated in the experiment. Moreover, the rapid change with position of the temperature signal adds to the difficulty of a quantitative comparison between theory and experiment. It is thus not surprising that only certain qualitative features are captured in the attempt of a comparison shown in Fig. 8.

\section{Discussion}

The interaction of traveling Rossby waves with spatially periodic modulations of the boundary gives rise to a quasiperiodic behavior in space as well as in time. The onset of convection is determined primarily by the local conditions as long as the modulation wavelength is large compared to the typical wavelength of Rossby waves. Pinning of convection in the form of the onset of a steady mode requires sufficiently high amplitudes of modulation and sufficiently high values of $\eta$ (see HB98), especially if the modulation wavelength is large. The onset of the steady mode could not be observed in the laboratory experiment because sufficiently high values of $\eta$ could not be reached. But the observed time depen- 


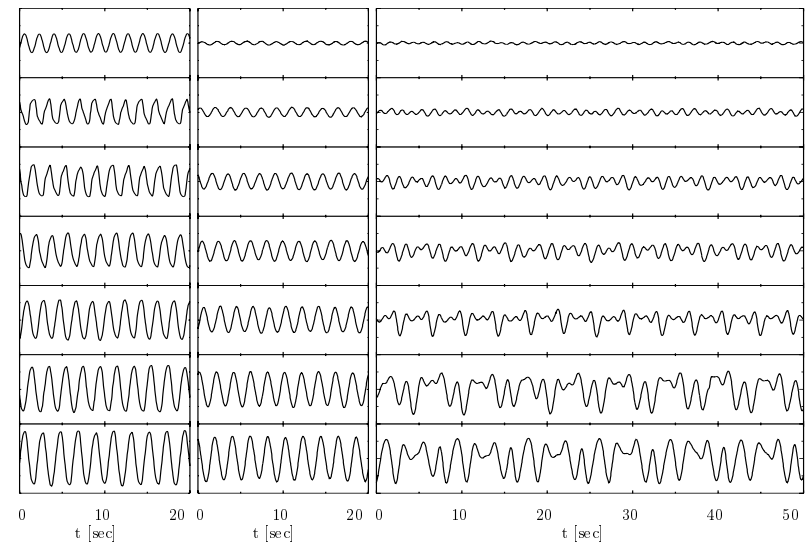

Fig. 7. Temperature close to the inner wall of the modulated cylindrical annulus as recorded by thermistors at the positions $y=$ $-6.1,-0.8$ and +0.6 (from left to right) in the case $\eta=458, \delta=$ $0.45, P=0.71$. The Rayleigh numbers (calculated without consideration of the convective contribution to the heat transport) are 9003, 10033, 11007, 12012, 12915, 13795 (from top to bottom).

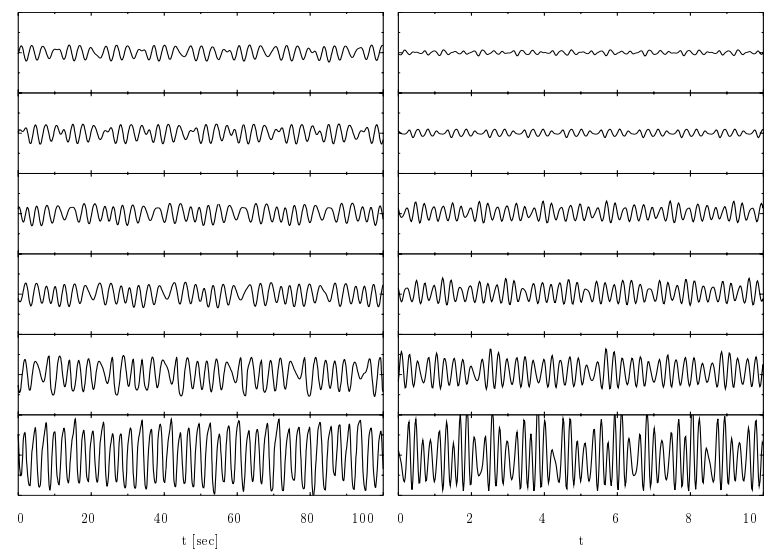

Fig. 8. Comparison of time dependences of temperatures measured by a thermistor at the position $y=4.7$ with temperature dependences at the same $y$-position $(x=-0.4)$ obtained from numerical simulations in the case $\eta=458, \delta=0.45, \alpha_{M}=0.296$. The Rayleigh numbers used in the simulations are 2385, 3500, 3600, 3900, 4000, 4250, 4500 (from top to bottom).

dence of the modulated thermal Rossby waves agrees semiquantitatively with the theoretical predictions.

The purely sinusoidal modulation studied in this paper has no direct geophysical application. It can be concluded, however, that the topography of the core-mantle boundary will affect convection in the form of thermal Rossby waves significantly only close to the virtual surface of the tangent cylinder which touches the inner core at its equator. At larger distances from the axis of rotation the geostrophic contours are not interrupted and dynamical features such as those studied in this paper can not be expected. Of course, Lorentz forces which have been neglected in the present study may also play a role in the Earth's core. But this topic should be addressed in another paper.
Acknowledgement. The support of the Deutsche Forschungsgemeinschaft for the research reported in this paper is gratefully acknowledged. The authors are also indebted to A. Busse for her help in the production of the figures.

\section{References}

Azouni, A., Bolton, E. W., and Busse, F. H.: Experimental study of convection columns in a rotating cylindrical annulus, Geophys. Astrophys. Fluid Dyn. 34, 301-317, 1986.

Bassom, A. P. and Soward, A. M.: Localized rotating convection induced by topography, Physica D 97, 29-44, 1996.

Bell, P. I.: The effects of bumps on convection in the Earth's core, $\mathrm{PhD}$ thesis, University of Newcastle upon Tyne, 1993.

Bell, P. I. and Soward, A. M.: The influence of surface topography on rotating convection, J. Fluid Mech. 313, 147-180, 1996.

Busse, F. H.: Asymptotic theory of convection in a rotating cylindrical annulus, J. Fluid Mech. 173, 545-556, 1986.

Busse, F. H. and Carrigan, C. R.: Convection induced by centrifugal buoyancy, J. Fluid Mech. 62, 579-592, 1974.

Busse, F. H., Hartung, G., Jaletzky, M., and Sommermann, G.: Thermal Convection Driven by Centrifugal Buoyancy in the Laboratory and its Applications to Planetary Problems, Dyn. Atmospheres Oceans 27, 161-174, 1997.

Hartung, G., Busse, F. H., and Rehberg, I.: Drifting convection rolls induced by spatial modulation, in: "Nonlinear Evolution of Spatio-Temporal Structures in Dissipative Continuous Systems", (Eds) Busse, F. H. and Kramer, L., Plenum Press, New York, 61-65, 1990.

Hartung, G., Busse, F. H., and Rehberg, I.: Time-dependent convection induced by broken spatial symmetry, Phys. Rev. Letts. 66, 2742-2745, 1991.

Herrmann, J. and Busse, F. H.: Stationary and time dependent convection in the rotating cylindrical annulus with modulated height, Phys. Fluids 10, 1611-1620, 1998.

Hide, R.: Fluctuations in the Earth's rotation and the topography of the core-mantle interface, Phil. Trans. R. Soc. Lond. A328, 351-363, 1989.

Jaletzky, M. and Busse, F. H.: New patterns in centrifugally driven thermal convection, Proc. Nat. Acad. Sci. 97, 5060-5064, 2000.

Kelly, R. E. and Pal, D.: Thermal convection with spatially periodic boundary condition: resonant wavelength excitation, J. Fluid Mech. 86, 433-456, 1978.

Pal, O. and Kelly, R. E.: Thermal convection with spatially periodic nonuniform heating: nonresonant wavelength excitation, in: "Heat Transfer 1978" (Proc. VI Int. Heat Transfer Conf.), 2, 235238, 1978.

Schnaubelt, M. and Busse, F. H.: Convection in a rotating cylindrical annulus. Part 3. Vacillating and spatially modulated flows, J. Fluid Mech. 245, 155-173, 1992.

Schnaubelt, M. and Busse, F. H.: Thermal Convection in the Rotating Cylindrical Annulus, Acta Astron. et Geophys. Univ. Comenianae 19, 63-78, 1997.

Soward, A. M. and Bassom, A. P.: Topographic rotating convection, Acta Astron. Geophys. Universitatis Comenianae 19, 277-292, 1997.

Zimmermann, W., Painter, B., and Behringer, R.: Pattern Formation in an Inhomogeneous Environment, in: "Evolution of Spontaneous Structures in Dissipative Continuous Systems", (Eds) Busse, F. H. and Müller, S. C., Springer, Lecture Notes in Physics, m55, 266-293, 1998. 